\title{
Trabajo remoto y liderazgo adaptativo en la gestión escolar en instituciones educativas
}

\author{
José Arturo Vásquez Benites \\ javasquezb@ucvvirtual.edu.pe \\ https://orcid.org/0000-0002-7731-410X \\ Jaidy Marilyn Chauca Rosales \\ jmchaucac@ucvvirtual.edu.pe \\ https://orcid.org//0000-0001-6152-9774 \\ Groberti Alfredo Medina Corcuera \\ grome@ucvvirtual.edu.pe \\ https://orcid.org/0000-0003-4035-157X \\ Universidad César Vallejo \\ Chimbote- Perú
}

\section{RESUMEN}

El presente trabajo se desarrolló con el objetivo de explicar en qué medida el trabajo remoto y el liderazgo adaptativo influyen en la calidad de gestión escolar de las instituciones educativas de Pallasca-Ancash, 2021, la metodología tuvo un enfoque cuantitativo, tipo básica de alcance explicativo y diseño no experimental de corte transversal, analizados mediante una regresión logística ordinal. Se trabajó con una población censal compuesta por 29 directores, la técnica empleada fue la encuesta, los instrumentos fueron 3 cuestionarios que midieron el trabajo remoto, el liderazgo adaptativo y la calidad de gestión escolar sometidos a las pruebas de validez y confiabilidad, obteniendo los siguientes resultados: Las significancias del ajuste global al modelo es de 0.068 y de la bondad de ajuste es de 0.647 , así como una influencia de $34,9 \%$ del trabajo remoto y el liderazgo adaptativo sobre la calidad de gestión escolar, no habiendo influencia significativa, salvo cuando el nivel de trabajo remoto es medio y la calidad educativa es regular con una significancia de 0,005 y 0,048 respectivamente. Concluyéndose que el trabajo remoto y el liderazgo adaptativo solo tienen influencia moderada sobre la gestión escolar, por ello, se aceptó la hipótesis nula y se descartó la de investigación.

Palabras clave: trabajo remoto; liderazgo adaptativo; gestión escolar; regresión logística. 


\title{
Remote work and adaptive leadership in school management in educational institutions in pallasca-ancash
}

\begin{abstract}
This research aims to explain how much the remote work and the adaptive leadership influence the quality of school management in the educational institutions in PallascaAncash, 2021. For this purpose, It was used the quantitative research methodology with non-experimental design and cross-sectional study, analysed by an ordinal logistic regression. We worked with the population of 29 principals, used the technique of the survey and the instruments were three questionnaires which measured the remote work, the adaptive leadership and the quality od school management according to the tests of validity and reliability and we obtained the following results: The significance of the global fit to the model is 0.068 and the goodness of fit is 0.647 , as well as an influence of $34.9 \%$ of remote work and adaptive leadership on the quality of school management, so there is no significant influence, except when the level of remote work is medium and the quality of school management is regular, we obtained a significance of 0.005 and 0.048 respectively. In conclusion, remote work and adaptive leadership only have a moderate influence on school management, therefore, the null hypothesis was accepted and the research hypothesis was rejected.
\end{abstract}

Keywords: remote work; adapttive leadership; school management; logistic regression

Artículo recibido: 15 noviembre. 2021 Aceptado para publicación: 10 diciembre 2021 Correspondencia: pepe arturo24@hotmail.com

Conflictos de Interés: Ninguna que declarar 


\section{INTRODUCCIÓN}

Este artículo aborda el tema del trabajo remoto, que raíz de la pandemia por el Covid 19 se implementó en muchos países, incluido el Perú como parte de las medidas en diversos aspectos para reducir el avance de la misma. y cómo este se vincula al campo educativo, el que según Vargas y Brito (2021) desde el inicio de la emergencia sanitaria a nivel global, se implementó para docentes, directivos y administrativos, dando continuidad al servicio educativo mediante una gestión escolar remota. En ese sentido en Perú, el Ministerio de Educación puso en marcha la estrategia “Aprendo en Casa" para brindando el servicio educativo en la modalidad a distancia mientras dure la emergencia sanitaria y aprobó las disposiciones para el trabajo remoto.

Sin embargo, esta fue una medida, a decir de Hertelendy, McNulty, Mitchell, Gutberg, Lassar, Durneva, \& Rapp (2021) mostró las falencias de los sistemas sociales como el educativo, los directores asumerieron el reto de implementar dicha estrategia, enfrentado dificultades de diversa índole poniendo a prueba su liderazgo. por los que Cucho (2020) manifiesta que al igual que en otros países, el sistema educativo peruano no estuvo preparado para implementar la educación a distancia mediante el trabajo remoto.

En Ancash y en la provincia de Pallasca en particular la experiencia fue similar, puesto que los directores al inicio no manejaban adecuadamente las TIC dificultándole realizar actividades de manera virtual, así como para establecer mecanismos de comunicación con sus docentes y superiores; por otro lado, la brecha de conectividad impidió una adecuada participación de estudiantes y familias, la distancia de las IIEE y el hogar de los directivos hacía que estos tengan mayores dificultades, ello en desmedro de su liderazgo, y sumando a las condiciones de trabajo remoto se puede suponer que como consecuencia, la gestión escolar se vea afectada. razones que llevan a plantearse la interrogante ¿En qué medida el trabajo remoto y el liderazgo adaptativo ejercen influencia en la calidad gestión escolar de las instituciones educativas de Pallasca-Ancash 2021?

En ese sentido, la investigación se justifica por su relevancia social al explicar el nivel de gestión escolar en función del trabajo remoto y liderazgo; una implicancia práctica al aportar información que servirá como diagnóstico para nuevas investigaciones, ya que no existía estudios al respecto y usarse en la toma de decisiones de las autoridades educativas en sus diversos niveles. Aporta al cuerpo del conocimiento científico por lo que tiene un valor teórico, también tiene una utilidad metodológica, porque se trata de una 
investigación explicativa con tres (3) variables, al mismo tiempo permitió construir y validar un cuestionario para medir de calidad de la gestión escolar alineado a los estándares del SINEACE y otro para medir el nivel de liderazgo adaptativo.

Frente a ello, el objetivo de investigación busca "Explicar en qué medida el trabajo remoto y el liderazgo adaptativo ejercen influencia en la calidad gestión escolar de las instituciones educativas de Pallasca-Ancash, 2021". Teniendo como hipótesis de investigación (Hi) que "El trabajo remoto y el liderazgo adaptativo influyen significativamente en la gestión escolar de instituciones educativas de Pallasca-Ancash 2021; y su correspondiente hipótesis nula (H0): El trabajo remoto y el liderazgo adaptativo no influyen significativamente en la calidad gestión escolar de instituciones educativas de Pallasca-Ancash 2021.

Existen estudios previos relacionados a las variables comprendidas en la presente investigación. A nivel internacional se encontró estudios como el de Prasertcharoensuk, y Puttarakulwisan (2014) sobre la relación y el efecto de la cultura organizacional asociada con la gestión escolar de suficiencia en 100 escuelas secundaria de Tailandia, utilizó un cuestionario de cinco escalas Likert. Sus resultados muestran a ambas variables en un nivel alto, en el que existe relación positiva y significativa entre ellas con un nivel de significancia de 0.05 . Además, la cultura organizacional aporta en un 74,30\% de variación en el nivel de implementación en la gestión escolar de suficiencia. También Maya, Aldana e Isea en el 2019 en un estudio no experimental correlacional transaccional referente a Liderazgo Directivo y Educación de Calidad, con un cuestionario aplicado a una subdirectora, una coordinadora y diez profesores, llegaron a la conclusión que existe una correlación positiva baja, esto significa que el liderazgo directivo tiene incidencia directa en una educación de calidad, siendo este un liderazgo directivo democrático, efectivo, participativo y asertivo que involucre activamente al personal docente en los planes de trabajo, hace que la organización camine hacia la calidad.

A nivel nacional, Sardon (2017) realizó una investigación de diseño no experimental transeccional referido a liderazgo transformacional y gestión escolar en IIEE primarias, con 118 docentes de la ciudad de Ilave, utilizando 2 cuestionarios; concluyó que existe una correlación positiva moderada para dichas variables en todas sus dimensiones, de la misma manera el $48 \%$ de docentes, tiene una percepción de un buen nivel de liderazgo transformacional del directivo. Así también Guarniz y Rodriguez (2018) investigaron el 
liderazgo y la calidad de gestión educativa de las IIEE del nivel primaria en 37 directores y profesores, utilizando un cuestionario de 82 items; concluyeron que el liderazgo que ejercen los directivos de una IE es de suma importancia para lograr la calidad, ya que este influye en todos los factores de la calidad educativa.

También, Parraguez (2018), en un estudio descriptivo explicativo-propositivo, el liderazgo transformador y cultura organizacional en IIEE de secundaria" realizada con directores y docentes de IIEE de Santa Rosa - Chiclayo, utilizando un cuestionario y bajo la técnica hermenéutica, llegó a la conclusión que el liderazgo transformacional conlleva a los colaboradores a convertirse en personas creativas y comprometidas con el logro, identificadas con la organización, inspirando a los demás a seguirlo, pensando colectivamente, buscando metas significativas. Asimismo, Vicente (2020) en su estudio correlacional sobre trabajo remoto y gestión administrativa realizada con 31 docentes, con un cuestionario de instrumento; llegó a la conclusión que el trabajo remoto y la gestión administrativa tienen una correlación positiva media y Llatas (2021) en su estudio correlativo sobre gestión directiva y el trabajo remoto de los docentes en los centros de educación básica alternativa - Callao, 2021 con 117 docentes en base a información obtenido de la aplicación de 2 cuestionarios, concluyó que existe una relación directa significativa de un $54 \%$.

A nivel regional-local se tiene Sáenz (2018) con un estudio relacionado a liderazgo pedagógico directivo y la calidad de la gestión educativa en la IE N89008 de Chimbote. Con un cuestionario adaptado de Autoevaluación de la Calidad Educativa, IACE, y un cuestionario adaptado para Valorar la Capacidad de Liderazgo, con 22 docentes de primaria y secundaria; concluyó que existe correlación directa positiva significativa entre ambas variables, y que el liderazgo directivo y la calidad de la gestión educativa son percibidos como buenos. También está Vasquez (2017), con un estudio correlacional sobre liderazgo y clima organizacional en la I.E. N88049 de Cascajal Bajo, SantaAncash, con 40 docentes entre primaria y secundaria, aplicando una escala para medir el nivel de liderazgo y un cuestionario para medir el clima organizacional, llegó a la conclusión que existe correlación de significancia bilateral, indicando la existencia de una relación con influencia positiva moderada entre ambas variables.

Teóricamente, sobre el trabajo remoto, se puede decir que es un término que se acuñó por primera en la década de 1970 (Allen, Golden, y Shockley, 2015). Sin embargo, es con el 
descubrimiento del nuevo coronavirus - COVID-19- en Wuhan, China deviniendo en una pandemia que afectó a casi todos los países del mundo (Azubuike, Adegboye y Quadri, 2021) y permanecerá como uno de los eventos más impactantes y perturbadores del siglo XXI (Shakibaei, De Jong, Alpkökin, y Rashidi, 2021) lo que obligó a los gobiernos en todo el mundo a implementar medidas para evitar la propagación del COVID-19, siendo el confinamiento de las personas en sus hogares una de ellas, generando que muchas profesiones se volcaron al teletrabajo (Bojovic, Benavides y Soret, 2021). Entendido como la realización de actividades relacionadas con el trabajo de forma remota utilizando tecnologías de la información y la comunicación (Mouratidis y Papagiannakis, 2021).

En el Perú, mediante el D.U. 026-2026, se implementa el trabajo remoto, definiéndolo como: "la prestación de servicios subordinada con la presencia física del trabajador en su domicilio o lugar de aislamiento domiciliario, utilizando cualquier medio o mecanismo que posibilite realizar las labores fuera del centro de trabajo, siempre que la naturaleza de las labores lo permita" modalidad en la que entre otras cosas, advirtiéndole al trabajador de sus obligaciones y medidas o condiciones de seguridad y salud en el trabajo durante el trabajo remoto entre otros. Por otro lado, el trabajador quedaba obligado a cumplir las normas de seguridad de la información, confidencialidad y protección de los datos y la información proporcionada para la prestación de servicios, cumplir los protocolos de seguridad y salud en el trabajo, estar disponible, para las coordinaciones necesarias durante la jornada laboral. (El Peruano, 2020. 15 de marzo)

Como era natural, la implementación del trabajo remoto requiere de medios y equipos informáticos entendidos como medio digital, que puede ser un medio cuyo contenido se representa enviado en forma legible por máquina o cuyos métodos de producción y la difusión involucran tecnologías de la información de diversos tipos (Bateman, 2021) cuya calidad de la participación de los empleados en el trabajo remoto tendrá un impacto diferente en el rendimiento y responderá al estilo de liderazgo más apropiado a las interacciones masivas en canales digitales (Figueiredo, 2021)

Este estudio se asume la teoría del "liderazgo adaptativo de, definida como la "práctica de movilizar a las personas para que afronten desafíos difíciles y prosperen” (Heifetz, Grashow y Linsky, 2012) o redefinido por Torner (2020) como la "Aptitud para abordar, gestionar e influenciar durante un desafío adaptativo, sin precedentes, movilizando a las personas para que lo afronten individual y colectivamente"; además 
Corazzini y Anderson (2014) afirman que pone a las personas ante la posibilidad de enfrentar ciertos problemas que surgen dentro del trabajo, sean ellas mismas las que lo resuelvan por estar en contacto directo scon estos y no requieren de un alto grado de experticia, sino de contacto y observación.

El panorama actual del liderazgo educativo según Ackerman, Mette, y Biddle (2018) ofrece una gama de desafíos que poco se reconocen o aprecian, y que generan incertidumbre, por lo que Jayan, Wai y Musa (2016) afirman que el siglo XXI plantea una multitud de desafíos impredecibles frente a los conocimientos y prácticas existentes; Linsky y Lawrence (2011) agregan que los desafíos que enfrenta el liderazgo escolar del siglo XXI están íntimamente entrelazados con los desafíos más amplios y profundos que enfrenta la comunidad mundial, más allá de las cualidades distintivas de la educación.

Las dimensiones de esta variable se relacionan con lo que Heifetz, et. al. (2012) llama el triángulo básico de la observación, interpretación e intervención ante un problema o desafío, cuya definición y solución requiere de aprendizaje; para ellos existen 2 formas de entender y abordar los problemas por lo que en palabras de Torres y Arroyo (2020) es indispensable distinguir entre los problemas técnicos y los problemas adaptativos, ya que los problemas técnicos por más complejos e importantes que sean, sus soluciones son conocidas; mientras que según Madariaga (2020) en los desafíos adaptativos, el problema radica en los mismos colaboradores, por cuanto son sus pensamientos, valores y comportamientos los que deben cambiar para lograr el objetivo compartido, es decir, afrontar un desafío es propiciar el descubrimiento.

Abordar desafíos se basa en 3 procesos descritos por Heifetz, et. al. (2012) observar (abordar problemas), implica ver objetivamente el desafío desde diversas perspectivas, utilizando la metáfora "abandonar la pista de baile y subir al palco es una manera excelente de lograrlo". Interpretar (gestión del cambio), este proceso implica elaborar una explicación o hipótesis de lo observado, evaluando diversas alternativas de solución y diversos aspectos. Intervención (intervenciones efectivas), es implementar medidas para dar respuesta al problema o desafío, respondiendo a la interpretación incluyendo diversas perspectivas. Una buena intervención debe sacar de la zona de confort a los integrantes de la organización.

Centrando la mirada en el sector educativo y en especial a los directivos, creemos que los desafíos adaptativos como sistema de compromisos, valores y lealtades en competencia 
no han sido resueltos todavía, las acciones se centran más desde la autoridad tradicional, por lo que es urgente hacer que se cambie dichas prácticas hacia el abordaje de desafíos adaptativos. En ese sentido, el papel del director es clave para lograr mejores resultados ya que problemas de los colegios coinciden con las deficiencias de sus líderes (Mulford, 2006) por ello el tema del liderazgo cobra importancia; convirtiéndose en una de las preocupaciones centrales de las organizaciones educativas.

La tercera variable es gestión escolar, enmarcada en la sociedad actual se demanda cada vez más de mayor calidad en todos los campos, experimentando un proceso de crecimiento sin límites en lo que concierne al valor de la calidad, para Bodero (2014) lograr la calidad es una prioridad y al mismo tiempo un problema; y desde el campo educativo hablar de calidad educativa es mirar a la gestión escolar que realiza el director como líder. En ese sentido, Vargas y Brito (2021) gestión implica administrar y organizar procesos interconectados entre sus elementos, en lo que concierne a la gestión de la escuela pública depende del sistema educativo, de la gestión institucional y la gestión pedagógica adscritas a la organización ministerial.

Para el presente estudio, asumimos la gestión escolar como el "conjunto de acciones articuladas entre sí que emprende el equipo directivo en una escuela para promover y posibilitar la consecución de la intencionalidad pedagógica en y con la comunidad educativa" (Ministerio de Educación, 2014) en el documento "Marco del Buen Desempeño Directivo" en el que se viene impulsando un modelo de gestión escolar centrada en los aprendizajes, en ella el directivo debe crear un escenario propicio para lograr los aprendizajes fundamentales, deseables y necesarios. Buscando además responder al modelo de acreditación del Sistema Nacional de Evaluación, Acreditación y Certificación de la Calidad Educativa (SINEACE)

Tomando como cómo referencia el SINEACE (2016) las dimensiones las dimensiones que considera esta variable son: la Gestión Estratégica; procesos y prácticas de gestión referidas a mantener una visión compartida; La formación integral: esta dimensión se ocupa del aspecto central de la gestión escolar; procesos pedagógicos que aseguren la formación integral de los estudiantes; La tercera dimensión corresponde al soporte y recursos para los procesos pedagógicos, esto es poner en práctica acciones gestión para tener una infraestructura segura, saludable y accesible, que facilite los procesos de enseñanza y aprendizaje; La última dimensión se vincula a los resultados, es decir, el 
logro de competencias de acuerdo al perfil de egreso, es definir mecanismos para evaluar a los estudiantes al terminar la secundaria, la satisfacción de quienes reciben el servicio educativo y el seguimiento que se hace a los egresados.

\section{ESTRATEGIAS METODOLÓGICAS O MATERIALES Y MÉTODOS}

La investigación fue básica, porque busca producir conocimiento; bajo el enfoque cuantitativo de alcance explicativo porque su objetivo es explicar la ocurrencia de un fenómeno y la condiciones en qué se manifiesta. El diseño fue no experimental de corte transversal, analizados mediante una regresión logística ordinal. Cuyo diagrama se presenta a continuación:

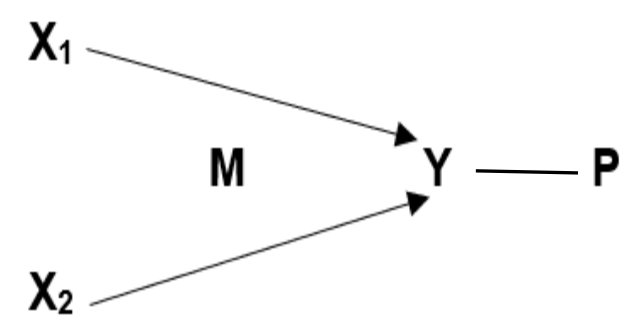

\section{Componentes:}

$\mathrm{M}=$ Muestra.

P $\quad \mathrm{X}_{1}$ y $\mathrm{X}_{2}=$ Variables independiente o influyentes.

$\mathrm{Y}=$ Variable dependiente $\mathrm{o}$ influida

$\mathrm{P}=$ Propuesta.

Se trabajó con tres variables, dos de ellas independientes o influyentes: Trabajo remoto, liderazgo adaptativo y una variable dependiente como es calidad gestión escolar, todas cuantitativas. La población comprometida fue de 29 directores cuyos criterios de inclusión que se trate de instituciones educativas de primaria, secundaria o ambos, así como que no tengan aula a cargo; los mismos que pasaron a formar parte de la muestra. Para el recojo de información se utilizó la técnica de la encuesta y como instrumentos 3 cuestionarios: "Cuestionario para medir el nivel desempeño en el trabajo remoto", de 25 items y cuyos de niveles de rango son Muy eficiente, eficiente, medio, Deficiente y Muy deficiente; "Cuestionario para medir el nivel de liderazgo adaptativo", con 30 items y niveles de rango: Muy alto, Alto, Medio, Bajo y Muy bajo y “Cuestionario para medir la calidad de gestión escolar" de 50 items, cuyos nivel de rango son de Muy buena, Buena, Regular, Mala y Muy mala. Validados mediante juicio de expertos y sometidos a la prueba de alfa de Cronbach para determinar su nivel de confiabilidad obteniendo resultados de $\alpha=0.860,0.980$ y 0.960 respectivamente.

El recojo de la información se hizo mediante formularios Google organizados en una base de datos, procesados estadísticamente utilizando el programa SPSS mediante la regresión logística ordinal, siguiendo a Garivay (2021) se siguió los siguientes pasos: 
Primero se validó el modelo mediante el ajuste global, luego la bondad de ajuste de los datos, el tercer paso fue el pseudo R2, para saber qué tanto por ciento las variables independientes explican la variable dependiente; Finalmente, se estimó los parámetros para explicar la influencia sobre la variable dependiente; pasos necesarios para contrastar la hipótesis.

\section{RESULTADOS Y DISCUSIÓN}

\subsection{Resultados}

\section{Análisis descriptivo}

\section{Tabla 1}

Resultados de la percepción de desempeño en el trabajo remoto por niveles

\begin{tabular}{|l|c|c|}
\hline & Frecuencia & Porcentaje \\
\hline Muy Deficiente & 0 & 0 \\
\hline Deficiente & 1 & 3,4 \\
\hline Medio & 11 & 37,9 \\
\hline Eficiente & 14 & 48,3 \\
\hline Muy Eficiente & 3 & 10,3 \\
\hline Total & 29 & 100,0 \\
\hline
\end{tabular}

Fuente: Base de datos del cuestionario para medir el nivel de desempeño en el trabajo remoto.

Interpretación: La tabla1 muestra los resultados de la variable trabajo remoto, en la que 14 directores se ubican en un nivel eficiente, siendo el 48,3\%, 11 en el nivel medio haciendo el 37,9\%; 3 en nivel muy eficiente representando un 10,3\% y solo 1 en nivel deficiente que representa el $3,4 \%$ de los 29 directores encuestados.

\begin{tabular}{|c|c|c|}
\hline \multicolumn{3}{|c|}{$\begin{array}{l}\text { Tabla } 2 \\
\text { Resultados del desarrollo de liderazgo adaptativo por Niveles }\end{array}$} \\
\hline & Frecuencia & Porcentaje \\
\hline Muy bajo & 0 & 0 \\
\hline Bajo & 0 & 0 \\
\hline Medio & 4 & 13,8 \\
\hline Alto & 11 & 37,9 \\
\hline Muy alto & 14 & 48,3 \\
\hline Total & 29 & 100,0 \\
\hline
\end{tabular}

Fuente: Base de datos del cuestionario para medir el nivel de desarrollo de liderazgo adaptativo, 
Interpretación: La tabla 2 refleja el nivel en que directores encuestados consideran que ha desarrollado el liderazgo adaptativo, de ellos 14 se ubican en el nivel muy alto $(48,3 \%)$, 11 perciben un nivel alto $(37,9 \%)$, mientras los 4 restantes en el nivel medio $(13,8 \%)$ y ninguno en los niveles de bajo y muy bajo.

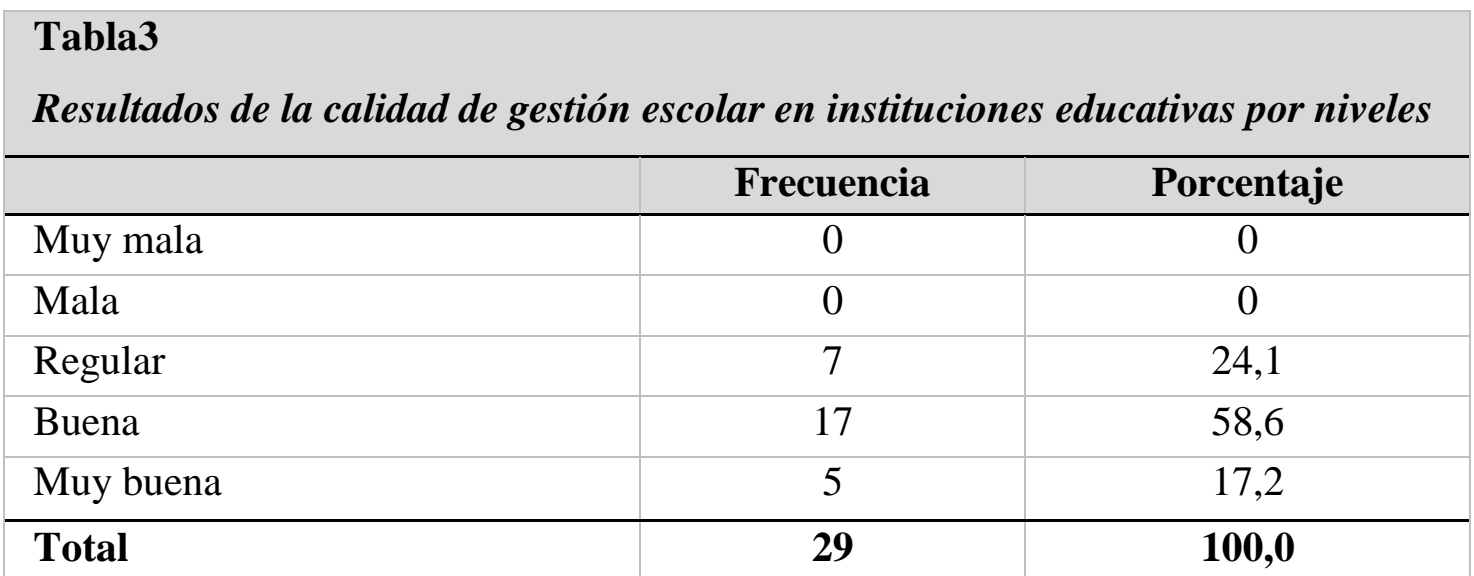

Fuente: Base de datos del cuestionario para medir el nivel de calidad de gestión escolar.

Interpretación: La tabla 4 muestra los resultados de la variable dependiente, gestión escolar; aquí se apreciar que 17, que equivale al 58,6\% se ubica en el nivel bueno; el siguiente nivel es el regular con 7 encuestados, haciendo un $24,1 \%$ y 5 consideran que la calidad de gestión escolar de sus IE es muy buena, esta cantidad representa el 17,2\%; asimismo, no hay ninguno en los niveles malo y muy malo.

\section{Análisis Inferencial}

\section{Tabla 4}

Información del ajuste de los modelos

\begin{tabular}{|l|c|c|c|c|}
\hline \multicolumn{1}{|c|}{ Modelo } & $\begin{array}{c}\text { Logaritmo de la } \\
\text { verosimilitud -2 }\end{array}$ & Chi-cuadrado & gl & Sig. \\
\hline Sólo intersección & 33,170 & & & \\
\hline Final & 22,904 & 10,267 & 5 & $\mathbf{0 6 8}$ \\
\hline
\end{tabular}

Fuente: Base de datos SPSS

Interpretación: Antes de la prueba de hipótesis, son necesarios los supuestos previos. El primero de ellos es la validación del modelo mediante el ajuste global, para saber si las variables introducidas explican el modelo; para ello el p-valor de significancia bilateral debe ser menor a a0,05. Sin embargo, como puede observarse en tabla 4 dicho valor es de 0.068 lo que quiere decir que el trabajo remoto y liderazgo adaptativo no tendrían influencia significativa en la calidad de gestión escolar. 


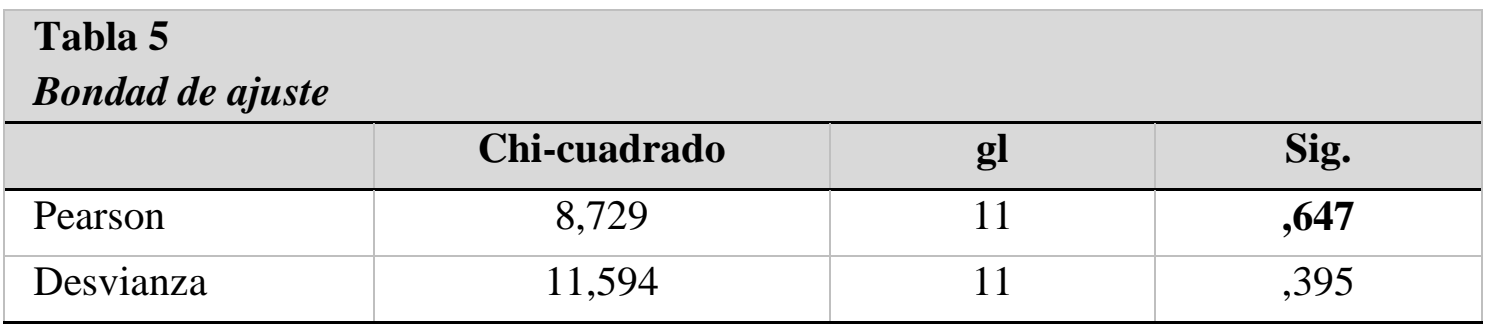

Fuente: Base de datos SPSS

Interpretación: El segundo resultado es la bondad de ajuste para comprobar si los datos observados son incompatibles con el modelo ajustado. Para tener la certeza de ello, tomando como referencia la significancia de Pearson, el p-valor debe ser mayor de 0.05 , para este caso en la tabla 5 se aprecia que la bondad de ajuste tiene significancia de 0.647 , dato que permite decir que el modelo sí se ajusta adecuadamente a los datos procesados.

\begin{tabular}{|l|l|}
\hline Tabla 6 & \\
Pseudo $\boldsymbol{R}$ cuadrado &, 298 \\
\hline Cox y Snell &, 349 \\
\hline Nagelkerke &, 185 \\
\hline McFadden & \\
\hline
\end{tabular}

Fuente: Base de datos SPSS

Interpretación: El tercer paso es la prueba de pseudo $\mathrm{R}^{2}$, para saber qué tanto por ciento las variables independientes trabajo remoto y liderazgo adaptativo explican la calidad de gestión escolar; para ello el valor debe ser $1(0 \leq \mathrm{R} 2 \geq 1)$. De la tabla 6 se toma el valor del coeficiente de determinación de Nagelkerke que es de 0,349 que al multiplicase por $100 \%$ se obtiene la influencia; esto significa que el trabajo remoto y liderazgo adaptativo, influyen la calidad de gestión escolar en un 34,9\%.

\begin{tabular}{|c|c|c|}
\hline \multicolumn{3}{|c|}{$\begin{array}{l}\text { Tabla } 7 \\
\text { Estimaciones de parámetro para las variables }\end{array}$} \\
\hline & & Sig. \\
\hline \multirow{2}{*}{ Umbral } & [Gestión Escolar = Regular] & ,005 \\
\hline & [Gestión Escolar = Buena] & ,568 \\
\hline \multirow{7}{*}{ Ubicación } & [Trabajo Remoto = Deficiente $]$ & . \\
\hline & [Trabajo Remoto = Medio $]$ & 048 \\
\hline & [Trabajo Remoto = Eficiente $]$ & ,099 \\
\hline & [Trabajo Remoto = Muy eficiente $]$ & . \\
\hline & [Liderazgo Adaptativo = Medio $]$ & ,275 \\
\hline & [Liderazgo Adaptativo = Alto $]$ & ,991 \\
\hline & [Liderazgo Adaptativo = Muy alto $]$ & \\
\hline
\end{tabular}

Fuente: Base de datos SPSS 
Interpretación: La tabla 7 muestra los resultados del último paso previo a la contrastación de hipótesis; la estimación de parámetros para explicar cómo las variables independientes influyen en la variable dependiente según sus categorías en base los valores de significancia de las categorías de cada variable, la misma debe ser menor a 0,05, ante ello se observa, por lo que se observa que solamente cuando el trabajo remoto es medio, la calidad de gestión escolar es regular; existiendo en este caso influencia significativa, más no en las demás categorías de esta variable no en las de liderazgo adaptativo; por lo que no se consideran en el análisis.

Por las razones expuestas, se toma la decisión de aceptar la hipótesis nula, es decir, el trabajo remoto y el liderazgo adaptativo no influyen significativamente en la calidad gestión escolar de instituciones educativas de Pallasca-Ancash 2021 a pesar del porcentaje de influencia, el ajuste del modelo no es significativo y en la mayoría de los niveles en la estimación de parámetros tampoco son significativos.

\subsection{Discusión}

La investigación tuvo la finalidad de "explicar en qué medida el trabajo remoto y el liderazgo adaptativo ejercen influencia en la calidad de gestión escolar de las instituciones educativas de Pallasca-Ancash, 2021”. Al cual se predecía una influencia significativa de las variables independientes sobre la variable dependiente. Al respecto, la tabla 2 muestra que trabajo remoto presenta que los directores están en un nivel eficiente con el 48,3\%, cifra menor al 50\%, siendo el nivel medio el más próximo con un porcentaje 37,9\%. Lo que se puede interpretar que los más significativos son estos niveles. Mientras que en liderazgo adaptativo las cifras tienen un comportamiento diferente, la tabla 2 muestra que son los niveles más altos los que prevalecen; el 48,3\% está en el nivel muy alto y el 37,9\% en nivel alto, haciendo que respectivamente predominen estos niveles. Y la calidad de gestión escolar, tal como lo muestra la tabla 3, el 58,6\% de directores identifican el nivel de calidad de su gestión como buena, seguido de una cuarta parte en regular. Haciendo que el nivel de gestión escolar marcadamente sea bueno.

Si bien cierto existe influencia del 34,9\%, lo que según Garivay (2021) podría considerarse influencia significativa teniendo en cuenta que para las Ciencias Sociales se considera significativo desde $30 \%$ a más. Sin embargo, el nivel de significancia del ajuste global es de 0,068 (mayor a 0,005) lo que indica que el trabajo remoto y liderazgo adaptativo no tendrían una influencia significativa sobre la calidad de Gestión escolar, 
considerándose influencia moderada; así como la estimación de parámetros solo muestra influencia significativa del nivel de trabajo remoto medio sobre el nivel de calidad de gestión escolar regular; lo que llevó a tomar la decisión de aceptar la hipótesis nula que significa que el trabajo remoto y el liderazgo adaptativo no ejercen influencia significativa en la calidad de gestión escolar en las IIEE de Pallasca, 2021.

comparándolo con otras investigaciones, encontramos por ejemplo a Vicente (2020) afirma que el trabajo remoto y la gestión administrativa tienen una correlación positiva media, ciertamente estos datos respaldan los resultados obtenido puesto que, individualmente si existe una influencia significativa del trabajo remoto en la gestión escolar sobre todo cuando ambas variables se ubican en un nivel intermedio.

En cuanto a la variable liderazgo, si bien cierto el presente estudio asume dicha variable como liderazgo adaptativo, no implica que sea un tipo de liderazgo diametralmente opuesto a otras propuestas de medir el liderazgo como el transformacional, por ejemplo. En ese sentido los resultados aquí obtenidos son respaldados por otros estudios, como también otros muestran diferencias. Partiendo de la idea que se ha encontrado influencia poco significativa con un porcentaje que no supera el $30 \%$ al medir dicha variable individualmente se tiene:

Estudios que de alguna manera respaldan los resultados de esta investigación como el de Vasquez (2017), quien habla de la existencia de una relación con influencia positiva moderada entre el estilo de liderazgo directivo sobre el clima organizacional, entendido este último como aspecto de la gestión escolar, igual que Sardon (2017) quien concluye que existe una correlación positiva moderada para el liderazgo transformacional y la gestión escolar en todas sus dimensiones, en el que la visión del liderazgo es buena, pero tal como aquí se aprecia no necesariamente lleva a una influencia de una sobre otra; también Maya, Aldana e Isea (2019) afirman la existencia de una correlación positiva pero baja entre liderazgo directivo y educación de calidad.

En el otro aspecto, Prasertcharoensuk, y Puttarakulwisan (2014) estudiaron la relación y el efecto de la cultura organizacional asociada con la gestión escolar de suficiencia encontrando una relación positiva y significativa en un nivel alto, en el que además la cultura organizacional influye en un $74,30 \%$ en la gestión escolar de suficiencia. Llatas (2021) afirma que la gestión directiva y el trabajo remoto tienen una relación significativa de 54\%; Sáenz (2018) también encontró una correlación directa positiva significativa 
entre liderazgo directivo y la calidad de la gestión educativa; para Guarniz y Rodriguez (2018) el liderazgo que ejercen los directivos de una IE es de suma importancia para lograr la calidad, ya que este influye en todos los factores de la calidad educativa y Parraguez (2018), concluye que el liderazgo transformacional conlleva a los colaboradores a convertirse en personas creativas y comprometidas con el logro, identificadas con la organización, inspirando a los demás a seguirlo, pensando colectivamente, buscando metas significativas; se asume que dichas características deberían reflejarse en un nivel de calidad de gestión escolar. Sin embargo, a la luz de los resultados obtenidos no es así.

Es importante también analizar dichos resultados a la luz de la teoría. En tal sentido, en cuanto a trabajo remoto se puede afirmar que esta modalidad de trabajo en concordancia con Shakibaei, De Jong, Alpkökin, y Rashidi (2021) este se masificó producto de la pandemia, incluido el sector educativo peruano, quien en palabras de Cucho (2020) este no estuvo preparado para implementar la educación a distancia mediante el trabajo remoto, por lo que generó múltiples problemas, se entiende que dicho problemas afectan a la gestión escolar.

En cuanto al liderazgo adaptativo, parafraseando a Hefetz, entendida como la aptitud para abordar, gestionar e influenciar en un desafío adaptativo (Torner 2020), es decir una forma diferente de abordar los problemas, con soluciones no conocidas; las que se entiende mejorarían en este caso la calidad de la gestión escolar, puesto ésta permanentemente se enfrenta a una serie de desafíos adaptativos y aunque los hallazgos muestren que los directores encuestados identifican que han desarrollado niveles muy alto y alto respectivamente de las habilidades de este tipo de liderazgo, no se está reflejando en la gestión escolar, ya que el nivel de significancia e influencia es bajo. Por lo que es necesario en todo caso centrar los esfuerzos para que ese alto nivel de liderazgo adaptativo se vea reflejado en la calidad de gestión escolar.

En relación a la gestión escolar, entendida como el "conjunto de acciones articuladas entre sí que emprende el equipo directivo en una escuela para promover y posibilitar la consecución de la intencionalidad pedagógica en y con la comunidad educativa" (Ministerio de Educación, 2014) prácticas que sin duda mejorarían la calidad de la educativa. Bodero (2014) reconoce que lograr la calidad es una prioridad y al mismo tiempo un problema, al cual hay que centrar la mirada y buscar los factores que podrían 
estar influyendo en la misma, cobrando sentido lo afirmado por Vargas y Brito (2021) que la gestión implica administrar y organizar procesos interconectados, es decir, una serie de aspectos como la gestión estratégica centrada en la planificación coherente; formación integral centrada en los aspectos pedagógicos; la gestión de los recursos financieros como materiales pedagógicos al servicio del aprendizaje y gestión de los resultados proyectando y volviendo al mismo tiempo la mirada hacia los egresados. En ese sentido, se deduce que requiere un liderazgo directivo al servicio de la mejora de todos estos aspectos en su integridad y articulación de los mismos.

\section{CONCLUSIONES}

Tomando como referencia el objetivo de la investigación; se concluye que el trabajo remoto y el liderazgo adaptativo tienen influencia moderada sobre la gestión escolar, acetándose la hipótesis nula y por ende se descartó la hipótesis de investigación. Dado que en los resultados se obtuvo que las significancias del ajuste global al modelo son de 0.068 y de la bondad de ajuste es de 0.647 , así como una influencia de 34,9\% del trabajo remoto y el liderazgo adaptativo sobre la calidad de gestión escolar, no habiendo influencia significativa, salvo cuando el nivel de trabajo remoto es medio y la calidad educativa es regular con una significancia de 0,005 y 0,048 respectivamente.

\section{LISTA DE REFERENCIAS}

Allen, T., Golden, T. y Shockley, M. (2015). How effective is telecommuting? Assessing the status of our scientific findings. Psychological Science in the Public Interest, 16(2), 40-68. Recuperado de: https://doi.org/10.1177/1529100615593273

Ackerman, R., Mette, I. \& Biddle, C. (2018). The Adaptive Challenges of Leadership in Maine Schools. Maine Policy Review, 27(1), 35-43. Recuperado de https://digitalcommons.library.umaine.edu/mpr/vol27/iss1/12/

Azubuike, O., Adegboye, O. \& Quadri, H. (2021), Who gets to learn in a pandemic? Exploring the digital divide in remote learning during the COVID-19 pandemic in Nigeria. International Journal of Educational Research Open, 2(2) 100022. Recuperado de https://doi.org/10.1016/j.ijedro.2020.100022.

Bateman, J. (2021) What are digital media?, Discourse, Context \& Media, 41, 100502, ISSN 2211-6958, https://doi.org/10.1016/j.dcm.2021.100502. Recuperado de (https://www.sciencedirect.com/science/article/pii/S2211695821000386) 
Bodero, H. (2014). El impacto de la calidad educativa. Apuntes. Revista de Ciencias Sociales, $\quad 4(01), \quad$ 112-117. $\quad$ Recuperado de https://dialnet.unirioja.es/servlet/articulo?codigo $=5042937$

Bojovic, D., Benavides, J., \& Soret, A. (2020). What we can learn from birdsong: Mainstreaming teleworking in a post-pandemic world. Earth System Governance, 5, 100074. Recuperado de https://doi.org/10.1016/j.esg.2020.100074

Corazzini, K. \& Anderson, R. (2014). Adaptive leadership and person-centered care: a new approach to solving problems. North Carolina Medical Journal, 75(5), 352354. Recuperado de doi: https://doi.org/10.18043/ncm.75.5.352

Cucho, E. (2020). Liderazgo directivo y desempeño docente en tiempos de pandemia en las instituciones educativas de Pucallpa [Tesis de maestría, Universidad Los Angeles de Chimbote]. Repositorio de la ULADECH, recuperado de http://repositorio.uladech.edu.pe/handle/123456789/20627

Figueiredo, J. (2021). The challenges of digital leadership. Revista de Administração Contemporânea, 25(4), e210043. Recuperado de https://doi.org/10.1590/19827849rac2021210043.

Garivay, J. (30 de Junio de 2021). Procesos Metodológicos y análisis de datos para el diseño no experimental: técnicas multivariables. [Archivo de video]: Recuperado de https://www.youtube.com/watch?v=hOAh_C_n4w

Guarniz, R. y Rodriguez, S. (2018). El Liderazgo en la calidad de Gestión Educativa de las Instituciones Educativas de Primaria del Distrito de Asunción, Cajamarca 2018 [Tesis de maestría, UCV). Repositorio de la UCV, recuperado de https://hdl.handle.net/20.500.12692/28288

Hertelendy, A. McNulty, E., Mitchell, C., Gutberg, J., Lassar, W., Durneva, P. \& Rapp, D. (2021). Crisis leadership: The new imperative for MBA curricula, The International Journal of Management Education, 19(3), 1-8. Recuperado de https://www.sciencedirect.com/science/article/pii/S1472811721000835

Jayan, M., Wai, K. \& Musa, K. (2016) Investigating the Relationship of Adaptive Leadership and Leadership Capabilities on Leadership Effectiveness in Sarawak Schools. Procedia - Social and Behavioral Sciences, 224, 540-545, recuperado de https://doi.org/10.1016/j.sbspro.2016.05.433. 
Llatas, T. (2021). Gestión directiva y trabajo remoto de los docentes en los centros de educación básica alternativa - Callao, 2021. [Tesis de grado, UCV] Recuperado de Ucv.edu.pe. https://hdl.handle.net/20.500.12692/70105

Maya, E. Aldana, J. \& Isea, J. (2019). Liderazgo Directivo y Educación de Calidad. Revista Interdisciplinaria de Humanidades, Educación, Ciencia y Tecnología, 5(9), 114-129. Recuperado de https://doi.org/10.35381/cm.v5i9.102

Ministerio de Educación (2014) Marco del buen desempeño directivo. Lima: MINEDU. Director (e) de la Dirección General de Desarrollo de las Instituciones Educativas. Recuperado de http://www.minedu.gob.pe/n/xtras/marco_buen_desempeno_directivo.pdf

Mouratidis, K. \& Papagiannakis, A. (2021) COVID-19, internet, and mobility: The rise of telework, telehealth, e-learning, and e-shopping. Sustainable Cities and Society, 103182. Recuperado de https://doi.org/10.1016/j.scs.2021.103182

Parraguez, L. (2018). Propuesta teórica de liderazgo transformador y mejora de cultura organizacional en instituciones educativas de educación secundaria. EDUCARE ET COMUNICARE, 6(2), 19-31. Recuperado de https://doi.org/10.35383/educare.v2i11.201

Perú. Sistema Nacional de Evaluación y Acreditación de Calidad Educativa - SINEACE. (2016). Modelo de Acreditación para instituciones de Educación Básica. Lima: SINEACE. Recuperado de https://www.sineace.gob.pe/wpcontent/uploads/2017/01/Resoluci\%C3\%B3n-N\%C2\%B0-242-2016-SINEACECDAH-P-Se-aprueba-el-Modelo-de-Acreditaci\%C3\%B3n-para-instituciones-deEducaci\%C3\%B3n-B\%C3\%A1sica-resoluci\%C3\%B3n-anexo.pdf

Prasertcharoensuk, T. \& Puttarakulwisan, B. (2014). Relationship between Organization Culture and Sufficiency School Management, Procedia - Social and Behavioral Sciences, 116, 796-801, //doi.org/10.1016/j.sbspro.2014.01.300. Recuperado de https://www.sciencedirect.com/science/article/pii/S1877042814003176

Sáenz, L. (2018). Liderazgo pedagógico directivo y la calidad de la Gestión Educativa de la Institución Educativa N 89008 , Chimbote, 2017 [Tesis de maestría, Universidad San Pedro] Repositorio de la Universidad San Pedro, Chimbote. Recuperado de http://repositorio.usanpedro.edu.pe/handle/USANPEDRO/10760 
Sardon, D. (2017), Liderazgo transformacional y gestión Escolar en instituciones educativas. Rev. Investig. Altoandin, 19 (3), 295 - 304. Recuperado de: http://dx.doi.org/10.18271/ria.2017.294

Shakibaei, S., De Jong, G., Alpkökin, P. \& Rashidi, T. (2021). Impact of the COVID-19 pandemic on travel behavior in Istanbul: A panel data analysis. Sustainable cities and society, 65, $102619 . \quad$ Recuperado de https://doi.org/10.1016/j.scs.2020.102619

Torner, C. (2020). Pandemia Covid 19 y liderazgo adaptativo. Importancia de su relación en una organización del sector eléctrico colombiano. Cuadernos Latinoamericanos de Administración. 16(31), 1-19. Recuperado de recuperado de https://doi.org/10.18270/cuaderlam.v16i31.3209

Torres, F. \& Arroyo, L. (2020). El liderazgo adaptativo en la práctica del derecho. Revista de Derecho Público, (92), 101-117. Recuperado de https://ultimadecada.uchile.cl/index.php/RDPU/article/view/57710/61863

Vargas, D. \& Brito, J. (2021). La gestión y el liderazgo escolar como factores de la diversidad cultural en la escuela en condiciones de emergencia sanitaria. Maestro y Sociedad, 18(2), 650-673. Recuperado de https://maestroysociedad.uo.edu.cu/index.php/MyS/article/view/5365/4990?fbcli d=IwAR1kdwIZGnb_qGQ8Q-54KXMBTWkEsR9BkFA1iUNLH7mBEbmLFy8g6_35Nw

Vásquez, E. (2017). Relación entre nivel de liderazgo y clima organizacional en la Institución Educativa N 88049 Cascajal Bajo, Provincia de Santa, Ancash 2016 [Tesis de Maestría, de la Universidad San Pedro] Universidad San Pedro. Chimbote. Recuperado de http://repositorio.usanpedro.edu.pe/handle/USANPEDRO/5534

Vicente, J. (2020) Trabajo remoto y gestión administrativa en la Institución Educativa Rosa Dominga Perez Liendo - La Victoria 2020 [Tesis de maestría, Universidad Cesar Vallejo] Repositorio de la Universidad Cesar Vallejo. Lima. Recuperado de https://hdl.handle.net/20.500.12692/49927 